\title{
Determinação de elementos essenciais maiores e traço em queijos por espectrometria de emissão atômica com plasma de argônio induzido após digestão parcial
}

\author{
Determination of minerals and trace elements in cheese by inductively-coupled
} plasma optical emission spectrometry after partial digestion

\author{
Carmen Silvia KIRA ${ }^{1 *}$, Vera Akiko MAIHARA ${ }^{2}$
}

\begin{abstract}
Resumo
Concentrações de $\mathrm{Ca}, \mathrm{Cr}, \mathrm{Cu}, \mathrm{Fe}, \mathrm{K}, \mathrm{Mg}, \mathrm{Mn}, \mathrm{Na}, \mathrm{P} \mathrm{e} \mathrm{Zn}$ foram determinadas em amostras de quatro tipos de queijo (mussarela, minas, prato e parmesão) por espectrometria de emissão atômica com plasma de argônio acoplado indutivamente após dissolução parcial (hidrólise com $\mathrm{HCl}$ ) e total da matéria orgânica (digestão por via seca). As concentrações da maioria dos elementos essenciais maiores e traço, usando o método de dissolução parcial, foram consideradas estatisticamente iguais às obtidas com o método de dissolução total da matéria orgânica, demonstrando a potencialidade da digestão parcial em análises de rotina.

Palavras-chave: derivados lácteos; pré-tratamento; ICP OES; minerais; análise multielementar.
\end{abstract}

\begin{abstract}
Concentrations of $\mathrm{Ca}, \mathrm{Cr}, \mathrm{Cu}, \mathrm{Fe}, \mathrm{K}, \mathrm{Mg}, \mathrm{Mn}, \mathrm{Na}, \mathrm{P}$ and $\mathrm{Zn}$ in four different types of cheese (mozzarella, white cheese, prato and parmesan) were analyzed by inductively-coupled plasma optical emission spectrometry (ICP-OES) after partial digestion (HCl hydrolysis) and total digestion of organic matter (dry ashing). The results obtained for most of the elements analyzed here by the partial digestion (HCl hydrolysis) and dry ashing methods were found to be statistically congruent, demonstrating the promising potential of the partial digestion method in routine analyses.

Keywords: dairy products; pretreatment; ICP OES; minerals; multielement analysis.
\end{abstract}

\section{Introdução}

Para que nosso organismo possa crescer e se manter é necessário que a alimentação seja balanceada. Leite e seus derivados são alimentos que se destacam pelo seu valor nutricional e dentre estes, os queijos estão ocupando um espaço cada vez maior no mercado brasileiro, porém as informações referentes ao seu conteúdo mineral são restritas. Dados da ABIA (Associação Brasileira das Indústrias de Alimentação) em relação aos alimentos industrializados mostraram que o queijo sofreu uma variação de consumo de mais de $51 \%$ entre os anos de 1994 e $1997^{19}$.

Queijo é basicamente uma fonte concentrada de nutrientes presentes no leite do qual ele é feito ${ }^{14,15}$. É extremamente difícil apresentar valores médios de nutrientes para queijos como um todo devido às diferenças nos processos de fabricação e padrões de identidade. Mesmo dentro de uma variedade de queijo, variações no tipo e composição de leite, processo, estação do ano e localidade podem levar a intensas flutuações na composição nutricional ${ }^{6,7,10,14,17}$.

Além de ser uma boa fonte de cálcio, o queijo também contém outros minerais, tais como magnésio e fósforo em quantidades apreciáveis, contribuindo assim significativamente para a ingestão diária recomendada para esses elementos ${ }^{20}$.

Recebido para publicação em 3/3/2006

Aceito para publicação em 19/7/2007 (001683)

Instituto Adolfo Lutz, Divisão de Bromatologia e Química,

Av. Dr. Arnaldo, 355, CEP 01246-902, São Paulo - SP, Brasil,

E-mail: carmkira@ial.sp.gov.br

Instituto de Pesquisas Energéticas e Nucleares - IPEN

Laboratório de Análise por Ativação, CEP 05508-000, São Paulo - SP, Brasil,

E-mail:vmaihara@ipen.br

* A quem a correspondência deve ser enviada
Há muitas categorias de queijos, desde frescos a maturados e aqueles classificados segundo o conteúdo de matéria gorda e umidade ${ }^{4}$.

Minerais ou elementos essenciais maiores são elementos inorgânicos amplamente distribuídos na natureza e que realizam uma variedade expressiva de funções metabólicas no organismo humano; estes são necessários em quantidades variáveis, por causa da quantidade relativamente grande dos principais minerais ( $\mathrm{Ca}, \mathrm{Mg}, \mathrm{Na}, \mathrm{K}, \mathrm{P}$ ) e da quantidade excessivamente pequena dos elementos traço nos alimentos ${ }^{22}$.

Dessa maneira, torna-se fundamental o desenvolvimento de metodologias que permitam verificar a qualidade da composição mineral desse alimento (queijo), sob o ponto de vista nutricional e toxicológico.

A técnica de espectrometria de emissão atômica com plasma de argônio acoplado indutivamente (ICP OES) tem sido largamente empregada na determinação de elementos traço em alimentos, como o leite, pois se conseguem baixos limites de detecção, faixa linear de trabalho ampla e a possibilidade de se fazer determinações simultâneas multielementares ${ }^{3,5,7}$.

Geralmente as análises por métodos espectroscópicos necessitam uma preparação de amostra, que é considerado um passo crítico já que é responsável pela possível contribuição de erros sistemáticos, além de ser a etapa mais demorada numa análise ${ }^{11}$. Assim, há a necessidade em se estudar métodos de dissolução para amostras de leite e derivados, que sejam mais simples e rápidos para a determinação de elementos essenciais maiores e traço.

A escolha do procedimento de digestão depende da natureza da matriz, dos elementos a serem determinados e dos métodos instrumentais a serem usados em sua determinação ${ }^{12}$. 
Segundo ALEIXO e NÓBREGA ${ }^{1}$, a determinação de elementos essenciais traço em leite não é uma tarefa fácil devido à complexidade desta matriz. Na literatura, encontramos relatos sobre o uso de diferentes procedimentos de tratamento de amostra, tais como digestão por via seca, digestão por microondas, digestão a alta pressão e até procedimentos de análise direta do leite. Alguns desses procedimentos possuem a desvantagem de necessitar longos períodos de digestão ou apresentar algumas dificuldades na determinação devido à presença de gordura na composição do leite, que pode afetar a nebulização pneumática no ICP OES ou então afetar as condições de excitação no plasma (ICP OES) devido à formação de carbono residual, quando a amostra de leite é introduzida sem prévia digestão. Um método baseado na hidrólise da amostra com uso de $\mathrm{HCl}$ não apresentou essas desvantagens e foi utilizado com sucesso na determinação de minerais e elementos essenciais traço em amostras de leite ${ }^{13}$.

Uma vez que queijos são derivados lácteos cuja matriz apresenta gordura em sua constituição, a determinação de minerais e elementos essenciais traço torna-se bastante demorada utilizando-se o procedimento de digestão por via seca, podendo levar a perdas e/ou contaminações da amostra. Assim, foi aplicado o procedimento de digestão parcial, com hidrólise de $\mathrm{HCl}$, como pré-tratamento da amostra para queijos de diferentes tipos (mussarela, minas, prato e parmesão) para a determinação de elementos essenciais maiores e traço e os resultados comparados aos obtidos com a digestão por via seca.

\section{Material e métodos}

\subsection{Equipamentos}

\section{Instrumentação para a técnica de ICP OES}

Forno mufla; chapa elétrica; balança analítica; espectrômetro de emissão atômica com plasma de argônio acoplado indutivamente, modelo Optima 3000 DV, marca Perkin Elmer. As análises dos elementos essenciais maiores e traço em amostras de queijo foram realizadas neste equipamento; e os comprimentos de onda selecionados foram os seguintes: Ca 422,673 nm; Cr 267,716 nm; Cu 327,396 nm; Fe 259,940 nm; K 766,491 nm; Mg 280,270 nm; Mn 257,610 nm; Na 589,59nm; P 213,618 nm; Zn 213,856 nm.

\subsection{Vidraria}

Toda a vidraria utilizada foi descontaminada quimicamente

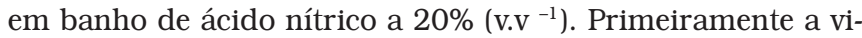
draria foi lavada com sabão neutro e água. Depois do enxágüe, a vidraria foi colocada em banho de ácido nítrico a $20 \%\left(\mathrm{v} . \mathrm{v}^{-1}\right)$ por 24 horas. Este procedimento foi repetido mais duas vezes e, em seguida, a vidraria foi enxaguada com água destilada e desionizada e seca em estufa.

\subsection{Reagentes}

Água destilada e deionizada com resistividade específica de $18,2 \mathrm{M} \Omega . \mathrm{cm}^{-1}$; ácido clorídrico $37 \%$ com teor reduzido de metais (Merck, Darmstadt, Germany); ácido clorídrico diluído na proporção 1:1 (foram misturados volumes iguais de $\mathrm{HCl} 37 \%$ e água destilada e deionizada); ácido nítrico 65\% P. A. (Merck, Darmstadt, Germany); solução-padrão estoque de Ca, Fe, K, $\mathrm{Na}, \mathrm{Mg}, \mathrm{P}$ e Zn - 10,00 \pm 0,03 mg. $\mathrm{mL}^{-1}$ (High Purity Standards); solução-padrão estoque de $\mathrm{Cr}, \mathrm{Cu}$ e $\mathrm{Mn}-1,000 \pm 0,002$ g.L $\mathrm{L}^{-1}$ (Merck, Titrisol). As soluções-padrão multielementares foram feitas em $\mathrm{HCl} 10 \%$ (v.v ${ }^{-1}$ ). A curva de calibração consistiu de cinco pontos mais o branco e as concentrações compreenderam as seguintes faixas: 2,5 a $100,0 \mathrm{mg} . \mathrm{L}^{-1}$ para $\mathrm{Ca}, \mathrm{K}$, Na e P; 0,25 a 10,0 mg. $\mathrm{L}^{-1}$ para Fe e Zn; 0,025 a 1,0 mg. $\mathrm{L}^{-1}$ para Cr; 0,125 a 5,0 mg. $\mathrm{L}^{-1}$ para $\mathrm{Cu} ; 0,625$ a $25,0 \mathrm{mg} . \mathrm{L}^{-1}$ para $\mathrm{Mg}$ e 0,0125 a 0,5 mg. $\mathrm{L}^{-1}$ para $\mathrm{Mn}$.

\subsection{Amostras}

Foram analisados quatro tipos de queijo: mussarela, minas, prato e parmesão. Cerca de $1 \mathrm{~kg}$ de cada tipo foi adquirido em supermercados da cidade de São Paulo.

\subsection{Liofilização das amostras}

Para a liofilização, as amostras de queijo mussarela, minas, prato e parmesão foram previamente trituradas em multiprocessador cujas lâminas eram de titânio; em seguida, foram colocadas em bandejas e espalhadas em finas camadas, congeladas por 24 horas e levadas ao liofilizador, modelo 30P2 - Edwards, por cerca de 40 horas. Depois da liofilização, as amostras foram trituradas e acondicionadas em sacos plásticos, previamente descontaminados com solução diluída de ácido nítrico e enxaguados com água destilada e desionizada.

\subsection{Pré-tratamento das amostras}

\section{Hidrólise com $\mathrm{HCl}$}

À cerca de $1 \mathrm{~g}$ da amostra liofilizada, pesado em erlemmeyer de $125 \mathrm{~mL}$, foram adicionados $10,0 \mathrm{~mL}$ de uma mistura de ácido clorídrico e água (1:1), que foi tampada com vidro de relógio pequeno. As amostras foram levadas à chapa elétrica a $100-150{ }^{\circ} \mathrm{C}$ e deixadas por 2 horas contadas após o início do refluxo. Ao final deste tempo, o digerido foi filtrado com papel de filtro quantitativo e o volume foi completado com água destilada e desionizada para balão volumétrico de $25,0 \mathrm{~mL}$. A seguir, a amostra foi submetida à leitura no ICP OES e fez-se um branco dos reagentes em paralelo.

\section{Digestão por via seca}

Pesaram-se cerca de $2 \mathrm{~g}$ da amostra liofilizada em cápsulas de porcelana que foram queimadas em bico de Bunsen e levadas à mufla a $450{ }^{\circ} \mathrm{C}$ para calcinar. Repetiu-se o processo até a destruição total da matéria orgânica. As cinzas das amostras foram então dissolvidas com $2,5 \mathrm{~mL}$ de $\mathrm{HCl}$ concentrado e transferidas quantitativamente para balão volumétrico de 25,0 mL com ajuda de água Milli-Q, para posterior leitura dos elementos de interesse no ICP OES.

\section{Análises estatísticas}

Para verificar se as médias obtidas entre os diferentes procedimentos de tratamento de amostra poderiam ser 
consideradas estatisticamente iguais, foi aplicado o teste de comparações múltiplas de Tukey-Kramer ${ }^{18,21}$.

Para aplicação dos testes estatísticos, foi utilizado o programa GraphPad Instat -versão 2.01, 1990-1993.

\section{Resultados e discussão}

Nas Tabelas 1 e 2, são apresentados os resultados das concentrações dos elementos determinados nas amostras liofilizadas dos queijos mussarela, minas, prato e parmesão, por ICP OES após a digestão total por via seca e hidrólise com $\mathrm{HCl}$. Os valores de concentrações referem-se às amostras de queijo in natura.

Com a hidrólise com $\mathrm{HCl}$, a amostra de queijo não foi levada à secura, uma vez que o erlemmeyer foi tampado com vidro de relógio, permitindo assim o refluxo; após as 2 horas de refluxo a dissolução da matéria orgânica obtida foi parcial.

Os resultados obtidos pela técnica de ICP OES nas amostras tratadas pelo método de dissolução parcial em chapa elétrica foram comparados com os resultados obtidos pelo método de digestão via seca, que é o método oficial para digestão de alimentos ${ }^{2}$.
Com a digestão por via seca, conseguiu-se a destruição total da matéria orgânica, que consumiu cerca de 24 horas.

Na digestão parcial em chapa elétrica, observou-se que a solução obtida após a filtração apresentava coloração marrom. Se a leitura não fosse realizada imediatamente após o preparo, ocorria decantação de um precipitado fino, contudo isso não afetou o resultado final. O método é bastante versátil, pois as leituras não precisaram ser feitas necessariamente no dia da preparação.

Na Tabela 3, são apresentados os resultados do teste estatístico para comparações entre as médias das amostras de queijo (mussarela, minas, prato e parmesão) pelos diferentes métodos de digestão (parcial e total).

Para as amostras de queijo, as concentrações dos elementos obtidas após a hidrólise com $\mathrm{HCl}$ foram concordantes com as concentrações determinadas após a digestão por via seca, para praticamente todos os elementos analisados. Exceção foi verificada para a amostra de queijo mussarela com relação ao cromo, cobre e fósforo, cujos teores determinados pelos diferentes tratamentos de amostra foram considerados estatisticamente diferentes. Exceção também foi verificada para a

Tabela 1. Resultados dos elementos determinados em amostras de queijo mussarela e minas por ICP OES, pelos métodos de digestão total e parcial (em mg.kg ${ }^{-1}$ ).

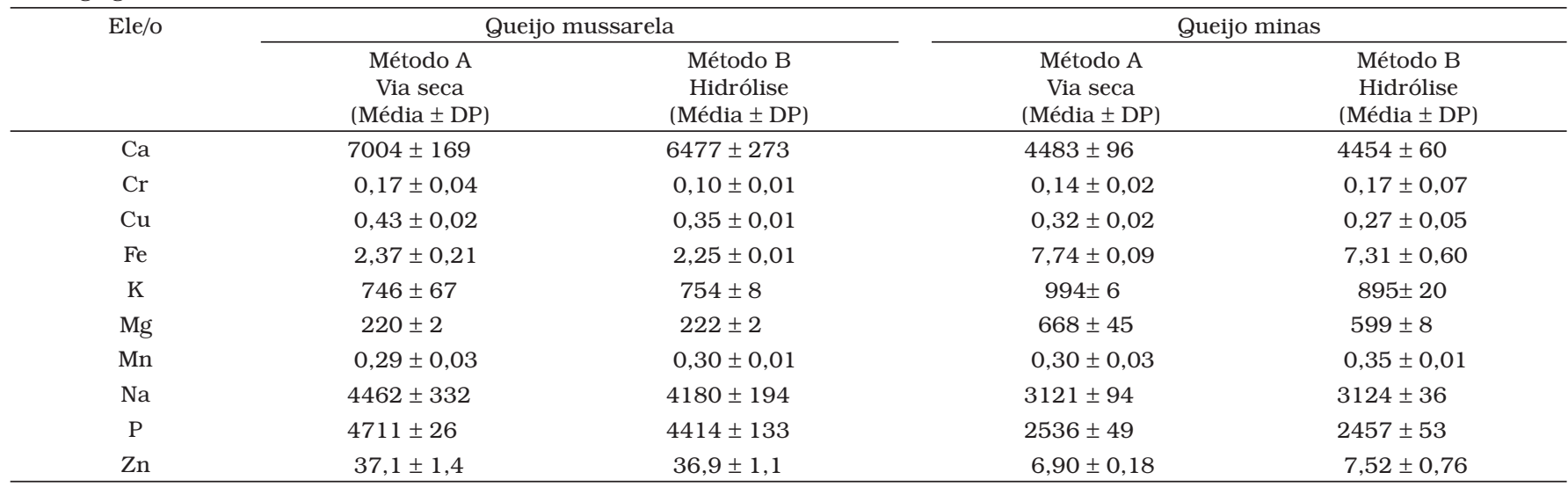

Número de determinações individuais: 3; e DP: desvio padrão.

Tabela 2. Resultados dos elementos determinados em amostras de queijo prato e parmesão por ICP OES, pelos métodos de digestão total e parcial (em mg.kg $\left.{ }^{-1}\right)$.

\begin{tabular}{|c|c|c|c|c|}
\hline \multirow[t]{2}{*}{ Ele/o } & \multicolumn{2}{|c|}{ Queijo prato } & \multicolumn{2}{|c|}{ Queijo parmesão } \\
\hline & $\begin{array}{c}\text { Método A } \\
\text { Via seca } \\
\text { (Média } \pm \text { DP) }\end{array}$ & $\begin{array}{c}\text { Método B } \\
\text { Hidrólise } \\
\text { (Média } \pm \text { DP) }\end{array}$ & $\begin{array}{c}\text { Método A } \\
\text { Via seca } \\
\text { (Média } \pm \text { DP) }\end{array}$ & $\begin{array}{c}\text { Método B } \\
\text { Hidrólise } \\
\text { (Média } \pm \text { DP) }\end{array}$ \\
\hline $\mathrm{Ca}$ & $7794 \pm 150$ & $7671 \pm 266$ & $10551 \pm 226$ & $10509 \pm 151$ \\
\hline $\mathrm{Cr}$ & $0,19 \pm 0,03$ & $0,21 \pm 0,02$ & $0,29 \pm 0,04$ & $0,29 \pm 0,02$ \\
\hline $\mathrm{Cu}$ & $0,45 \pm 0,03$ & $0,52 \pm 0,04$ & $0,31 \pm 0,03$ & $0,29 \pm 0,03$ \\
\hline $\mathrm{Fe}$ & $2,33 \pm 0,17$ & $2,30 \pm 0,10$ & $2,29 \pm 0,89$ & $2,43 \pm 0,48$ \\
\hline $\mathrm{K}$ & $878 \pm 50$ & $839 \pm 43$ & $989 \pm 38$ & $881 \pm 5$ \\
\hline $\mathrm{Mg}$ & $282 \pm 7$ & $268 \pm 11$ & $385 \pm 9$ & $395 \pm 10$ \\
\hline $\mathrm{Mn}$ & $0,24 \pm 0,02$ & $0,24 \pm 0,01$ & $0,26 \pm 0,07$ & $0,28 \pm 0,01$ \\
\hline $\mathrm{Na}$ & $5173 \pm 120$ & $5238 \pm 174$ & $10362 \pm 519$ & $10486 \pm 126$ \\
\hline $\mathrm{P}$ & $4835 \pm 96$ & $4663 \pm 182$ & $6732 \pm 153$ & $6657 \pm 85$ \\
\hline $\mathrm{Zn}$ & $32,2 \pm 1,6$ & $33,2 \pm 2,1$ & $48,4 \pm 1,2$ & $49,6 \pm 1,8$ \\
\hline
\end{tabular}

Número de determinações individuais: 3; e DP: desvio padrão. 
Tabela 3. Comparação entre as médias obtidas para as amostras de queijo mussarela, minas, prato e parmesão pela técnica de ICP OES, com diferentes métodos de digestão, aplicando o teste de Tukey-Kramer.

\begin{tabular}{ccccc}
\hline \multirow{2}{*}{ Ele/o } & Q. mussarela & Q. minas & Q. prato & Q. parmesão \\
\cline { 2 - 5 } & Métodos & Métodos & Métodos & Métodos \\
& A x B & A x B & A x B & A x B \\
\hline $\mathrm{Ca}$ & + & + & + & + \\
$\mathrm{Cr}$ & - & + & + & + \\
$\mathrm{Cu}$ & - & + & - & + \\
$\mathrm{Fe}$ & + & + & + & + \\
$\mathrm{K}$ & + & + & + & + \\
$\mathrm{Mg}$ & + & + & + & + \\
$\mathrm{Mn}$ & + & - & + & + \\
$\mathrm{Na}$ & + & + & + & + \\
$\mathrm{P}$ & - & + & + & + \\
$\mathrm{Zn}$ & + & + & + & + \\
\hline
\end{tabular}

A: Digestão via seca; B: Hidrólise com $\mathrm{HCl}$; +: aceita (as médias podem ser consideradas iguais com o nível de significância de $95 \%$ considerados); e -: rejeita (as médias não são consideradas iguais com o nível de significância de $95 \%$ considerados).

amostra de queijo minas, cujo teor de manganês diferiu estatisticamente entre os procedimentos de digestão da amostra. Para o queijo prato, verificou-se uma diferença estatisticamente significante para o cobre determinado utilizando-se os diferentes tratamentos de amostra.

As concentrações médias de cobre podem ser afetadas pelos diferentes conteúdos de gordura presentes na matriz, uma vez que o cobre está ligado aos glóbulos de gordura ${ }^{9}$. Segundo o Regulamento Técnico Geral para a fixação de Identidade e Qualidade dos Queijos, o queijo mussarela pode ser classificado como extragordo (conteúdo mínimo de matéria gorda de $60 \%$ ), gordo (conteúdo de matéria gorda entre 45,0 e $59,9 \%$ ) e semigordo (conteúdo de matéria gorda entre 25,0 a 44,9\%); o queijo minas como semigordo, o queijo prato como gordo e o queijo parmesão como semigordo ${ }^{4}$. Isto pode explicar as diferenças nas concentrações de cobre obtidas para os queijos mussarela e prato, os quais são constituídos por uma quantidade maior de matéria gorda em relação aos demais tipos de queijo analisados.

Segundo a literatura, a associação do fósforo com a caseína na presença de gordura pode dificultar sua liberação da matriz ${ }^{8,16}$. Isto pôde ser verificado pela menor concentração de fósforo obtida após a hidrólise com $\mathrm{HCl}$ em relação à concentração do elemento obtida após a digestão por via seca para a amostra de queijo mussarela.

Pode-se supor uma relação entre o teor de gordura e a associação de um elemento, como o cálcio, com a caseína, de forma mais intensa, dificultando a liberação do elemento da matriz, levando conseqüentemente a baixas recuperações.

Comparando-se os resultados obtidos para cálcio entre as diferentes matrizes analisadas, verifica-se que a maior variação na concentração deste elemento determinado por digestão via úmida em relação à concentração obtida por digestão via seca foi mais pronunciada para a amostra de queijo mussarela. Para as demais amostras, a variação nas concentrações de cálcio não foi superior a $2 \%$ pela digestão via seca e hidrólise com $\mathrm{HCl}$. A explicação para este fato pode ser devida à forte associação do cálcio com a caseína, pelo maior conteúdo de gordura presente na amostra de queijo mussarela em relação às demais amostras analisadas. Isso foi verificado no trabalho de De LA FUENTE e JUÁREZ ${ }^{8}$ que relataram que $2 / 3$ do cálcio estão associados com as micelas da caseína, que, na presença de gordura, causa dificuldades na determinação deste elemento em amostras de leite.

Os valores de desvios-padrão obtidos pelo método de digestão parcial em chapa elétrica foram comparativamente iguais e até menores para alguns elementos, como Ca, K, Mn, Na e P nas amostras de queijo parmesão (Tabelas 1 e 2).

Para verificar a exatidão e precisão dos métodos de digestão parcial e total, foram utilizados dois materiais de referência certificados (NIST 8435 Whole Milk Powder e NIST 1549 Non Fat Milk Powder). Pelos resultados obtidos, apresentados respectivamente nas Tabelas 4 e 5, verificou-se que as concentrações obtidas após a digestão total e parcial foram bastante concordantes com os valores de referência certificados para os elementos analisados.

Tabela 4. Resultados dos elementos determinados no material de referência certificado NIST8435 (Whole Milk Powder) por ICP OES, pelos métodos de digestão ( $\left.\mathrm{em} \mathrm{mg} \cdot \mathrm{kg}^{-1}\right)$.

\begin{tabular}{cccc}
\hline Ele/o & $\begin{array}{c}\text { Métodos de digestão } \\
\text { Determinação por ICP OES (Média } \pm \text { DP) }\end{array}$ & $\begin{array}{c}\text { Valor certificado } \\
\text { (Média } \pm \text { DP) }\end{array}$ \\
\cline { 2 - 3 } & $\begin{array}{c}\text { Método A } \\
\text { (Via seca) }\end{array}$ & $\begin{array}{c}\text { Método B } \\
(\text { Hidrólise com HCl) }\end{array}$ \\
\hline $\mathrm{Ca}$ & $9331 \pm 207(6)^{\mathrm{a}}$ & $9209 \pm 147(6)^{\mathrm{a}}$ & $9220 \pm 490$ \\
$\mathrm{Cu}$ & $0,44 \pm 0,05(6)^{\mathrm{a}}$ & $0,40 \pm 0,04(6)^{\mathrm{a}}$ & $0,46 \pm 0,08$ \\
$\mathrm{Fe}$ & $1,87 \pm 0,13(6)^{\mathrm{a}}$ & $1,95 \pm 0,43(6)^{\mathrm{a}}$ & $1,8 \pm 1,1$ \\
$\mathrm{~K}$ & $13844 \pm 263(6)^{\mathrm{a}}$ & $13484 \pm 288(6)^{\mathrm{a}}$ & $13630 \pm 470$ \\
$\mathrm{Mg}$ & $813 \pm 27(6)^{\mathrm{a}}$ & $794 \pm 17(6)^{\mathrm{a}}$ & $814 \pm 76$ \\
$\mathrm{Mn}$ & $0,17 \pm 0,02(6)^{\mathrm{a}}$ & $0,16 \pm 0,01(6)^{\mathrm{a}}$ & $0,17 \pm 0,05$ \\
$\mathrm{Na}$ & $3670 \pm 98(6)^{\mathrm{a}}$ & $3555 \pm 54(6)^{\mathrm{a}}$ & $3560 \pm 400$ \\
$\mathrm{P}$ & $7871 \pm 135(6)^{\mathrm{a}}$ & $7632 \pm 228(6)^{\mathrm{a}}$ & $7800 \pm 490$ \\
$\mathrm{Zn}$ & $27,7 \pm 2,0(6)^{\mathrm{a}}$ & $26,7 \pm 2,0(6)^{\mathrm{a}}$ & $28,0 \pm 3,1$ \\
\hline a: números de determinaçoes individuais; e DP: desvio padrão. &
\end{tabular}

a: números de determinações individuais; e DP: desvio padrão.

Tabela 5. Resultados dos elementos determinados no material de referência certificado NIST 1549 (Non Fat Milk Powder) por ICP OES, pelos métodos de digestão (em mg.kg $\left.{ }^{-1}\right)$.

\begin{tabular}{cccc}
\hline Ele/o & $\begin{array}{c}\text { Métodos de digestão } \\
\text { Determinação por ICP OES (Média } \pm \text { DP) }\end{array}$ & $\begin{array}{c}\text { Valor certificado } \\
\text { (Média } \pm \text { DP) }\end{array}$ \\
\cline { 2 - 3 } & $\begin{array}{c}\text { Método A } \\
\text { (Via seca) }\end{array}$ & $\begin{array}{c}\text { Método B } \\
\text { (Hidrólise com HCl) }\end{array}$ \\
\hline $\mathrm{Ca}$ & $13128 \pm 355(4)^{\mathrm{a}}$ & $13122 \pm 373(5)^{\mathrm{a}}$ & $13000 \pm 500$ \\
$\mathrm{Cu}$ & $0,66 \pm 0,07(5)^{\mathrm{a}}$ & $0,64 \pm 0,05(6)^{\mathrm{a}}$ & $0,7 \pm 0,1$ \\
$\mathrm{Fe}$ & $1,84 \pm 0,09(4)^{\mathrm{a}}$ & $1,75 \pm 0,29(5)^{\mathrm{a}}$ & $1,78 \pm 0,1$ \\
$\mathrm{~K}$ & $16881 \pm 495(5)^{\mathrm{a}}$ & $16807 \pm 721(6)^{\mathrm{a}}$ & $16900 \pm 300$ \\
$\mathrm{Mg}$ & $1149 \pm 48(6)^{\mathrm{a}}$ & $1103 \pm 10(6)^{\mathrm{a}}$ & $1200 \pm 30$ \\
$\mathrm{Mn}$ & $0,21 \pm 0,02(5)^{\mathrm{a}}$ & $0,22 \pm 0,02(6)^{\mathrm{a}}$ & $0,26 \pm 0,06$ \\
$\mathrm{Na}$ & $5139 \pm 232(4)^{\mathrm{a}}$ & $4990 \pm 242(6)^{\mathrm{a}}$ & $4970 \pm 100$ \\
$\mathrm{P}$ & $10603 \pm 374(6)^{\mathrm{a}}$ & $10117 \pm 138(4)^{\mathrm{a}}$ & $10600 \pm 200$ \\
$\mathrm{Zn}$ & $40,5 \pm 0,9(6)^{\mathrm{a}}$ & $40,4 \pm 2,8(6)^{\mathrm{a}}$ & $46,1 \pm 2,2$ \\
\hline a: números de determinacões individuais; e DP: desvio padrão. &
\end{tabular}




\section{Conclusões}

O método de digestão parcial adotado realizado em tempo relativamente curto ( 2 horas) foi adequado à análise multielementar simultânea de elementos essenciais maiores e traço em amostras de queijo, com boa exatidão e precisão. As concentrações obtidas para os elementos analisados após a hidrólise com $\mathrm{HCl}$ foram consideradas estatisticamente iguais às concentrações obtidas após a digestão total, com exceção de $\mathrm{Cr}$, Cu e $\mathrm{P}$ para o queijo mussarela, $\mathrm{Mn}$ para o queijo minas e $\mathrm{Cu}$ para o queijo prato. A causa para este fato pode ser atribuída à associação do elemento com a caseína, que, na presença de gordura, pode dificultar sua liberação da matriz. Em virtude da não necessidade de destruição total da matéria orgânica da amostra para a determinação de muitos elementos essenciais maiores e traço para as matrizes estudadas, diminuiu-se o tempo gasto no preparo de amostra e, portanto, o risco de contaminação.

\section{Referências bibliográficas}

1. ALEIXO, P. C.; NÓBREGA, J. A. Direct determination of iron and selenium in bovine milk by graphite furnace atomic absorption spectrometry. Food Chem., v. 83, n. 3, p. 457-462, 2003.

2. ASSOCIATION OF OFFICIAL ANALYTICAL CHEMISTS. Official methods of analysis of official analytical chemists. 15 ed. Washington, D. C., 1995, chap. 50, p. 13.

3. BIEGO, G. H. et al. Determination of mineral contents in different kinds of milk and estimation of dietary intake in infants. Food Addit. Contam., v. 15, n. 7, p. 775-781, 1998.

4. BRASIL. Portaria n. 146, de 07 de março de 1996. Regulamento técnico de identidade e qualidade de queijos. Diário Oficial da República Federativa do Brasil, Brasília, DF, 11 mar. 1996. Disponível em http:// www.agricultura.gov.br/sda/dipoa/portaria 146.htm. Acesso em: 24 set. 2002.

5. CARRIÓN, N. et al. Determination of calcium, potassium, magnesium, iron, copper and zinc in maternal milk by inductively coupled plasma atomic emission spectrometry. J. Anal. At. Spectrom., v. 9, n. 3, p. 205-207, 1994.

6. CICHOSCKI, A. J. et al. Characterization of Prato cheese, a Brazilian semihard cow variety: evolution of physico-chemical parameters and mineral composition during ripening. Food Control, v. 13, n. 4-5, p. 329-336, 2002.

7. CONI, E. et al. A methodological approach to the assessment of trace elements in milk and dairy products. Food Chem., v. 50, n. 2, p. 203-210, 1994.

8. DE LA FUENTE, M. A.; JUÁREZ, M. Rapid determination of calcium, magnesium, sodium and potassium in milk by flow atomic spectrometry after microwave oven digestion. Analyst, v. 120 , n. 1 , p. 107-111, 1995.
9. DE LA FUENTE, M. et al. Total and soluble contents of calcium, magnesium, phosphorus and zinc in yogurts. Food Chem., v. 80, n. 4 , p. $573-578,2003$.

10. FOX, P. F. Cheese: An overview. In: Cheese: chemistry, physics and microbiology. General aspects. Chapman \& Hall, 2. ed., v. 1, 1993, cap. 1, p. 1-25.

11. HOENING, M.; KERSABIEC, A-M. Sample preparation steps for analysis by atomic spectroscopy methods: present status. Spectrochim. Acta, part B, v. 51, n. 11, p. 1297-1307, 1996.

12. KARADJOVA, I. et al. Determination of $\mathrm{Cd}, \mathrm{Co}, \mathrm{Cr}, \mathrm{Cu}, \mathrm{Fe}, \mathrm{Ni}$ and $\mathrm{Pb}$ in milk, cheese and chocolate. Microchim. Acta, v. 134, n. 3-4, p. 185-191, 2000.

13. KIRA, C. S.; de MAIO, F. D.; MAIHARA, V. A. Comparison of partial digestion procedures for determination $\mathrm{Ca}, \mathrm{Cr}, \mathrm{Cu}, \mathrm{Fe}, \mathrm{K}, \mathrm{Mg}, \mathrm{Mn}$, $\mathrm{Na}, \mathrm{K}, \mathrm{P}$ and $\mathrm{Zn}$ in milk by inductively coupled plasma-optical emission spectrometry. J. AOAC Int., v. 87, n. 1, p. 151-156, 2004.

14. LANTE, A. et al. Content and characterization of minerals in milk in Crescenza and Squacquerone Italian fresh cheeses by ICP OES. Food Control, v. 17, n. 3, p. 229-233, 2006.

15. MENDIL, D. Mineral and trace metal levels in some cheese collected from Turkey. Food Chem., v. 96, n. 4, p. 532-537, 2006.

16. NEVILLE, M. C.; ZHANG, P.; ALLEN, J. C. Minerals, íons and trace elements in milk. Ionic interactions in milk. In: JENSEN, R. G. Handbook of Milk Composition. San Diego, California. Printed in the United States of America. Food Science and Technology International Series, Academic Press, 1995. cap. 7, p. 577-590, $919 \mathrm{p}$.

17. PEDRO, N. A. R.; de OlIVEIRA, E.; CADORE, S. Study of the mineral content of chocolate flavoured beverages. Food Chem., v. 95, n. 1, p. 94-100, 2006.

18. PITOCCHELLI, J. Multiple comparisons tests. [S. 1.]. 2001. Disponível em: <http://www.anselm.edu/homepage/jpitocch/ anova/multcomp. html>. Acesso em: 27 ago. 2002.

19. SECRETARIA DE COMUNICAÇÃO DO GOVERNO. Coleção de documentos da Presidência da República. In: Real: Quatro anos que mudaram o Brasil. Consumo e Qualidade de Vida. Disponível em: <http://www.planalto.gov.br/publi_04/COLECAO/PUBLICA. HTM>. Acesso em: 18 nov. 2005.

20. SPADOTI, L. M.; OLIVEIRA, A. J. Uso de leite reconstituído na fabricação de queijo mussarela. Ciênc. Tecnol. Aliment., v. 19, n. 1, p. 1-15, 1999.

21. VIEIRA, S. Teste $t$. Análise de Variância. In: Introdução à bioestatística. Rio de Janeiro, RJ: Editora Campus, 1981. cap. 12, p. 125-137. cap. 13, p. 141-155, 203 p.

22. WILliaMS, S. R. Minerals. In: Fundamentos de nutrição e dietoterapia. 6. ed. Porto Alegre: Artes Médicas, 1997. cap 8, p. 144-169. 664 p. Tradução: Regina Machado Garcez. 